Check for updates

Cite this: Chem. Sci., 2018, 9, 6779

๑ All publication charges for this article have been paid for by the Royal Society of Chemistry

Received 6th June 2018

Accepted 29th June 2018

DOI: $10.1039 / \mathrm{c} 8 \mathrm{sc} 02492 \mathrm{~h}$

rsc.li/chemical-science

\section{Interfacing porphyrins and carbon nanotubes through mechanical links $\uparrow$}

\author{
Leire de Juan-Fernández, $\ddagger^{\mathrm{a}}$ Peter W. Münich, $\ddagger^{\mathrm{b}}$ Arjun Puthiyedath, (D) Belén Nieto- \\ Ortega, (D) a Santiago Casado, ${ }^{a}$ Luisa Ruiz-González, ${ }^{C}$ Emilio M. Pérez (D) *a \\ and Dirk M. Guldi (iD *b
}

\begin{abstract}
We describe the synthesis of rotaxane-type species composed of macrocyclic porphyrin rings mechanically interlocked with SWCNT threads. The formation of mechanically interlocked SWCNTS (MINTs) proceeds with chiral selectivity, and was confirmed by spectroscopic and analytical techniques and adequate control experiments, and corroborated by high-resolution electron microscopy. From a thorough characterization of the MINTs through UV-vis-NIR absorption, fluorescence, Raman, and transient absorption spectroscopy we analyse in detail the electronic interactions of the porphyrins and the SWCNTs in the ground and excited states.
\end{abstract}

\section{Introduction}

The ability of metalloporphyrins to reversibly coordinate gases is exploited by nature in the transport of oxygen by the heme group. Directly related to this is another main biological function of porphyrinoid structures, that is, as co-factors in cytochrome P450, which is responsible for many catalytic redox reactions. Finally, the intense absorption in the visible region and the ability to accept electrons upon photoexcitation of the structurally related chlorophylls renders them ideal light antennas and primary electron acceptors in photosynthesis. Inspired by these crucial roles, porphyrins are one of the most extensively studied organic chromophores. ${ }^{1-5}$ A particularly active area of research is the combination of porphyrins and carbon nanotubes (CNT), which also benefits from the extraordinary physical properties of CNTs. ${ }^{6-9}$ Porphyrin-CNT hybrids have been successfully applied to the fabrication of bioinspired high-performance gas sensors, ${ }^{10,11}$ catalysts, ${ }^{12-15}$ model photosynthetic systems, ${ }^{\mathbf{1 6}-18}$ and other optoelectronic devices. ${ }^{19}$ The physical properties of all these artificial systems depend crucially on the nature of the linker between them.

A covalent porphyrin-CNT connection typically leads to good kinetic stability, and the structure of the spacer can be used to

\footnotetext{
${ }^{a}$ IMDEA Nanoscience, C/ Faraday 9, Ciudad Universitaria de Cantoblanco, 28049 Madrid, Spain. E-mail: emilio.perez@imdea.org

${ }^{b}$ Department of Chemistry and Pharmacy, Interdisciplinary Center for Molecular Materials, Friedrich-Alexander University of Erlangen-Nürnberg, Egerlandstr. 3, 91058 Erlangen, Germany. E-mail: guldi@fau.de

'Departamento de Química Inorgánica, Fac. C. C. Químicas, Universidad Complutense de Madrid, Avenida Complutense s/n, 28040 Madrid, Spain

$\dagger$ Electronic supplementary information (ESI) available. See DOI: $10.1039 / \mathrm{c} 8 \mathrm{sc} 02492 \mathrm{~h}$

\$ These authors contributed equally.
}

modulate porphyrin-CNT communication. ${ }^{20-22}$ Interfacing porphyrins and CNTs via non-covalent interactions provides an alternative means to generate porphyrin-CNT hybrids. ${ }^{23-29}$ The intrinsic porphyrin-CNT non-covalent interactions have been skillfully exploited to associate and purify single-walled CNTs (SWCNT) in a chirality and enantioselective fashion. ${ }^{\text {30-34 }}$

Mechanically interlocked molecules (MIM) are composed of covalent components linked together by their topology. Rotaxanes are prototypical examples of MIMs, in which one or more macrocycles are linked to a dumbbell-shaped linear component: the thread. The mechanical bond imparts MIMs with unique dynamic properties. ${ }^{35,36}$ Specifically, the macrocycles can be moved along or around the thread through controlled submolecular motion. This is a key step for the construction of some of the most advanced examples of synthetic molecular machines. ${ }^{37-39}$

We have developed methods to thread SWCNTs through macrocycles to form rotaxane-like mechanically interlocked nanotube derivatives (MINT) using a clipping strategy. ${ }^{\mathbf{4 0}}$ More recently, groups led by Kruss, ${ }^{41}$ and Miki and Ohe, ${ }^{42}$ have described the encapsulation of SWCNTs into peptide barrels and rigid cycloparaphenyleneacetylenes, respectively. The native structure of SWCNTs is preserved upon formation of MINTs, while the extreme aspect ratio of SWCNTs prevent the macrocycles from dissociating, providing MINTs with a kinetic stability comparable to that of covalently functionalized SWCNTs. ${ }^{4-45}$ We have also documented that MINTs feature unique physical properties, which are quite different from classic supramolecular SWCNT derivatives. ${ }^{\mathbf{4 6 , 4 7}}$

Here, we interface porphyrins and SWCNTs through mechanical links. The MINT-forming reaction proceeds with chiral selectivity for the smaller diameter SWCNTs. The electronic interactions between the porphyrins and the nanotubes 
in the ground state, as well as the charge separation and recombination processes and their underlying dynamics upon photoexcitation are also described in detail.

\section{Results and discussion}

Following experimental fine-tuning (see the ESI $\dagger$ ) we focused on a U-shaped molecule equipped with two porphyrin recognition units for SWCNTs (U-por in Fig. 1). A hexamethylene spacer provides flexibility to template the macrocyclization around SWCNTs through ring-closing metathesis (RCM) of the terminal alkenes, to form the corresponding MINTs. The synthesis and full characterization of U-por and mac-por is described in the ESI. $\dagger$

We explored the formation of MINT-por through templated RCM of U-por in the presence of $(6,5)$-enriched SWCNTs. The diameter of the latter is $0.75 \mathrm{~nm}$, which is a good fit for the cavity of mac-por, as shown by the energy-minimized molecular structure (HF-3c) ${ }^{48}$ shown in Fig. 1B. SWCNTs (2 mg) were suspended in tetrachloroethane (TCE, $2 \mathrm{~mL}$ ) through sonication and mixed with $\mathbf{U}$-por $(4.2 \mathrm{mM})$ and Grubbs' $2^{\text {nd }}$ generation catalyst at room temperature (rt) for 72 hours.

Next, the suspension was filtered through a polytetrafluoroethylene membrane with a pore size of $0.2 \mu \mathrm{m}$ and washed with dichloromethane (DCM) to remove non-threaded macrocycles, catalyst and any remaining linear precursors. Three resuspension-filtration iterations complete the purification. Hereafter, the samples were dried and subjected to thermogravimetric analysis (TGA) to quantify the degree of functionalization. TGA showed a weight loss of $24 \%$ at around $340{ }^{\circ} \mathrm{C}$, corresponding to the porphyrinic material. We performed experiments in which the concentration of $\mathbf{U}$-por was varied between 1.06 and $8.4 \mathrm{mM}$ to modulate the degree of functionalization. The product formed at the lowest

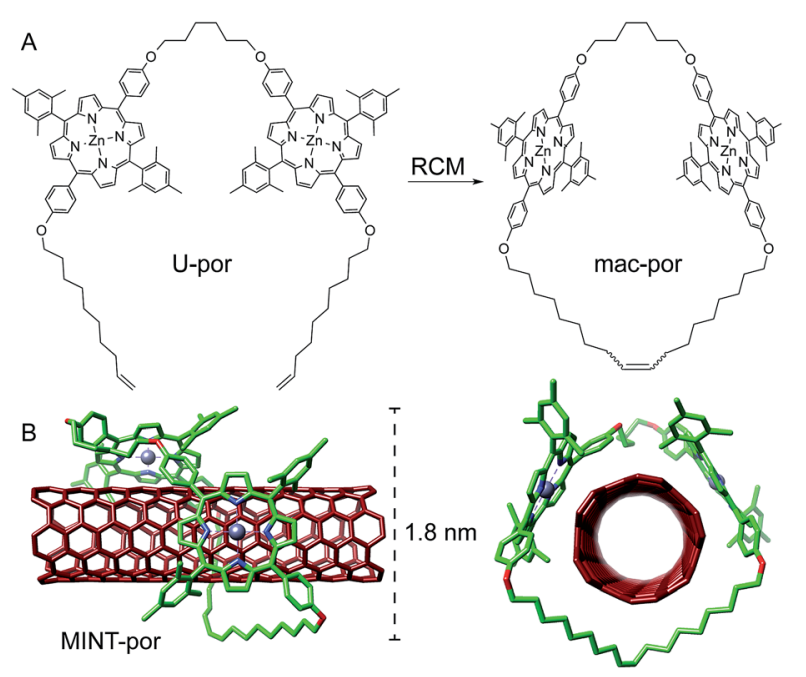

Fig. 1 (A) Chemical structures of U-por and mac-por. (B) Side and front view of the energy-minimized structure (HF-3c) of MINT-por, showing the diameter of the macrocycle. $C$ atoms in green (mac-por) or dark red (SWCNT), $\mathrm{N}$ in blue, $\mathrm{O}$ in red, $\mathrm{Zn}$ in metallic gray. $\mathrm{H}$ atoms omitted for clarity. concentration of the U-shaped precursor showed a loss of $10 \%$ at $340{ }^{\circ} \mathrm{C}$, whereas a weight loss of $26 \%$ was observed for the highest concentration (see the ESI $\dagger$ ). ${ }^{49}$ In addition, the derivative of the TGA data features a single peak for the porphyrinic material, confirming that oligomers of U-por adsorbed onto SWCNTs are absent in MINT-por. ${ }^{43,50,51}$

To test the stability of MINT-por, we heated the samples for 30 min under reflux in TCE $\left(\mathrm{bp}=147^{\circ} \mathrm{C}\right)$, followed by a thorough rinse with DCM. TGA of the resulting samples showed a subtle decrease of $3 \%$ in functionalization confirming the MINT stability.

Investigations of MINT-por by means of atomic force microscopy (AFM) and transmission electron microscopy (TEM) corroborate the presence of rotaxane-type species. Fig. 2A shows a typical AFM topographic image of a spin-casted TCE suspension of MINT-por. Individualized SWCNTs with heights around $0.8 \mathrm{~nm}$ decorated with objects, which are consistently around $2.0 \mathrm{~nm}$ high, are in sound agreement with mac-por around a SWCNT (Fig. 1B). Macrocycles adsorbed onto SWCNTs rather
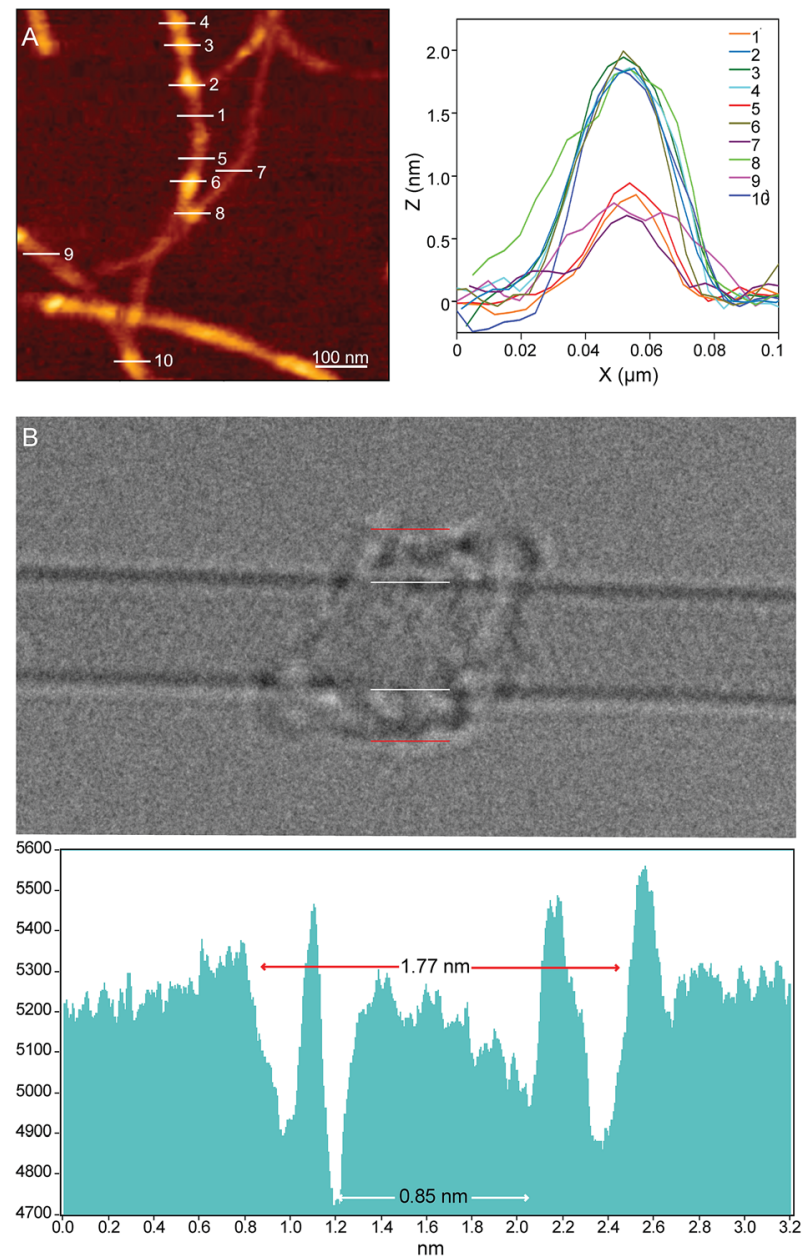

Fig. 2 (A) AFM topographic image of a suspension of MINT-por in TCE and corresponding height profiles along the lines marked in the topographic image. (B) AC-HRTEM image of an individual SWCNT functionalized by a single macrocycle in MINT-por and corresponding profiles (white lines and arrows mark SWNT diameter, red lines and arrows mark mac-por diameter). 
than threaded by SWCNTs are expected to show a significantly smaller height.

Aberration-corrected high resolution TEM (AC-HRTEM) images further confirm the rotaxane-like structure of MINTpor. They were drop-casted from an isopropanol suspension onto copper grids and reveal mostly bundled SWCNTs with functionalized sidewalls. These co-exist, however, with isolated SWCNTs of $0.8 \mathrm{~nm}$ diameter, in which single circular objects of a size of $c a .1 .8 \mathrm{~nm}$ are discernable. A representative AC-HRTEM micrograph of MINT-por with close to atomic resolution is shown in Fig. 2B, which shows a SWCNT with a diameter of $0.85 \mathrm{~nm}$, surrounded by a macrocycle that shows a diameter of $1.77 \mathrm{~nm}$. In contrast to our previous MINTs, in which we observed macrocycle-like rings around SWCNTs, ${ }^{13}$ the large dimensions of the porphyrins - in the range of 1.4-1.8 nm, comparable to the diameter of the macrocycle - confer the macrocycles a box-like shape. We note that the distances between the macrocycle and the SWCNT sidewalls correspond to very close van der Waals contacts $(<0.4 \mathrm{~nm})$. Finally, from the difference in contrast between the SWCNT walls we conclude that mac-por is threaded by SWCNTs rather than physisorbing on them. We note that, even under these low voltage conditions (see the ESI $\dagger$ ), the sample is eventually damaged under the ebeam, most likely starting with the more sensitive $\mathrm{C}-\mathrm{H}$ bonds of the alkyl chain, ${ }^{52}$ but eventually damaging even the SWCNT wall (see Fig. S2†).

To gain insights into the electronic properties of MINT-por, we carried out UV-vis-NIR, fluorescence, and Raman spectroscopy measurements. Steady state absorption spectra $\left(\mathrm{D}_{2} \mathrm{O} / \mathrm{SDBS}\right.$ $(1 \mathrm{wt} \%, \mathrm{rt}))$, of $(6,5)$-enriched SWCNTs (black) and the corresponding MINTs (red) are compared in Fig. 3A. In the MINT-por sample, the Soret-band absorption of the porphyrins is evident at $430 \mathrm{~nm}$. As far as the absorption spectra of $(6,5)$-enriched SWCNTs are concerned, $S_{22}$ transitions are noted in the visible region, while the $S_{11}$ transitions dominate the nIR region. In particular, $(6,5)$-SWCNT related maxima appear at 570 and $981 \mathrm{~nm}$. Features of $(7,5)$-SWCNTs are seen at 649 and $1022 \mathrm{~nm}$, whereas those of $(7,6)$-SWCNTs evolve at 664 and $1136 \mathrm{~nm}$. In MINT-por, all of the aforementioned features are red-shifted to 572, 982, 652, 1022, 664, and $1137 \mathrm{~nm}$, on one hand, and broadened, on the other hand. From the aforementioned we conclude weak electronic interactions between SWCNTs and the porphyrin in the ground state. When turning to the porphyrin absorption spectrum, the Soret-band absorption maximizes at $429 \mathrm{~nm}$ in DMF for MINT-por as well as for U-por Fig. S3. $\dagger^{53}$

To probe the chirality selectivity during the MINT formation, ${ }^{47}$ the relative absorption intensities in dispersions of pristine SWCNTs and MINT-por are inspected. A look at, for example, the $(6,5)$-SWCNTs features in the nIR region reveals that the relative intensity is higher for SWCNTs than for MINTpor. The trend is reversed for $(7,5)$-SWCNTs. Implicit is a chiral selectivity of the MINT formation based on different SWCNT diameters.

To shed light on the excited state interactions in MINT-por, the SWCNT fluorescence was analyzed in the nIR-region Fig. 3B and C. In spectra of SWCNT and MINT-por,
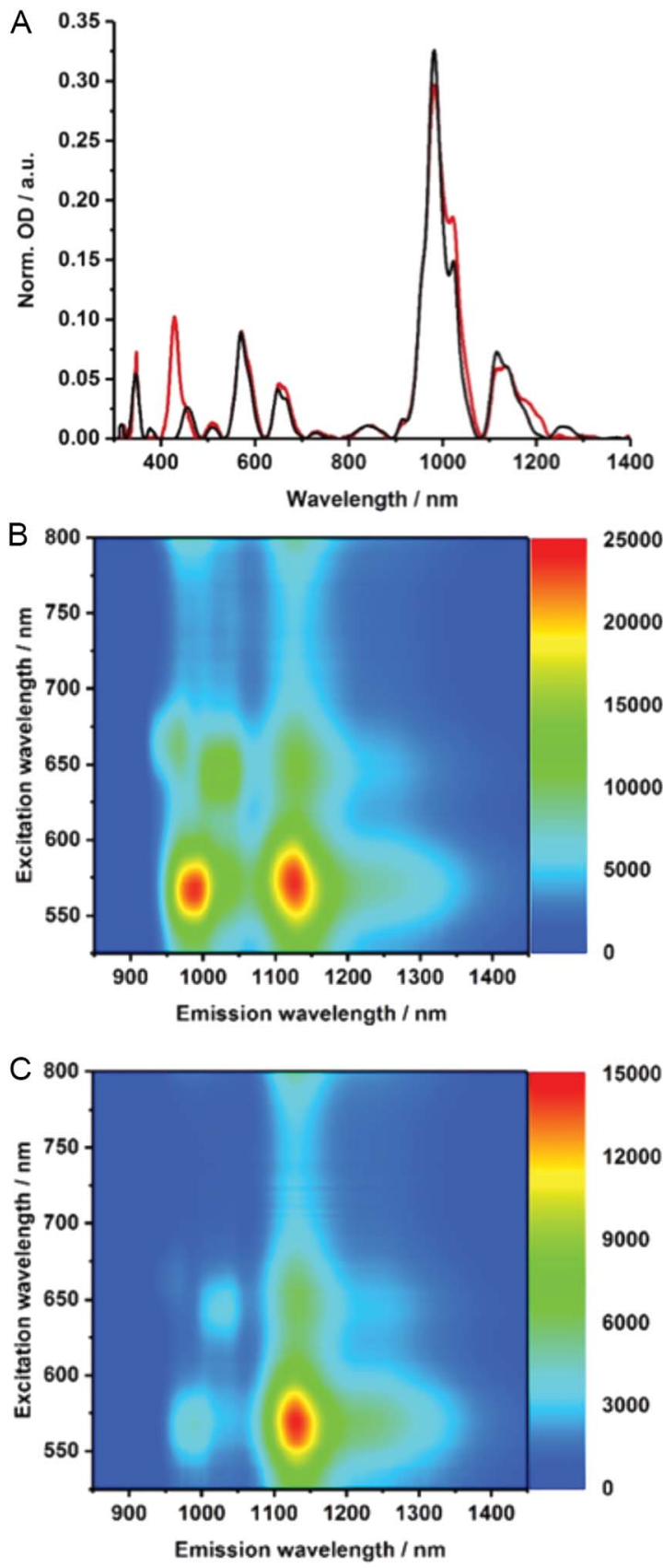

Fig. 3 (A) Normalized absorption spectra of $(6,5)$-enriched SWCNTs (black), and MINT-por (red) in $\mathrm{D}_{2} \mathrm{O} / \mathrm{SDBS}$ ( $1 \mathrm{wt} \%, \mathrm{rt}$ ), baseline-corrected and normalized to the $(7,6)-$ SWCNT absorption at $1136 \mathrm{~nm}$. 3D nIR fluorescence spectra of (B) (6,5)-enriched SWCNTs and (C) MINTpor in $\mathrm{D}_{2} \mathrm{O} / \mathrm{SDBS}(1 \mathrm{wt} \%$, rt) with OD of 0.21 at $570 \mathrm{~nm}$.

characteristic fluorescence features of $(8,3),(6,5),(7,5),(8,4)$ and $(7,6)$-SWCNTs are present. At first glance, the fluorescence features of pristine SWCNTs at 967, 988, 1031, 1127, and $1130 \mathrm{~nm}$ are - in line with the steady state absorption measurements - red-shifted in MINT-por; the new maxima are at 967, 993, 1034, 1130, and $1132 \mathrm{~nm}$. A closer look at the fluorescence spectra shown in Fig. $\mathrm{S} 4 \uparrow$ reveals quenching, which is stronger for small diameter SWCNTs, that is, $(8,3)$-, $(6,5)-$, and $(7,5)$-SWCNT, than for larger diameter SWCNTs, that 
is, $(8,4)$ - and $(7,6)$-SWCNT. A possible rationale is a higher degree of functionalization in the case of smaller diameter SWCNTs in comparison to their larger diameter analogues. Quenching, although moderate in nature, was also observed for the porphyrin fluorescence - Fig. S4. $\dagger$ Quenching in MINT-por is likely to stem from deactivation pathways such as energy or electron transfer, which compete with the fluorescent decay.

Electronic interactions as seen in the excited state might also impact the Raman features. To this end, about 1000 Raman spectra were taken for each sample - Fig. 4. They show small upshifts of the D-, G- and 2D-modes from 1298, 1584 and
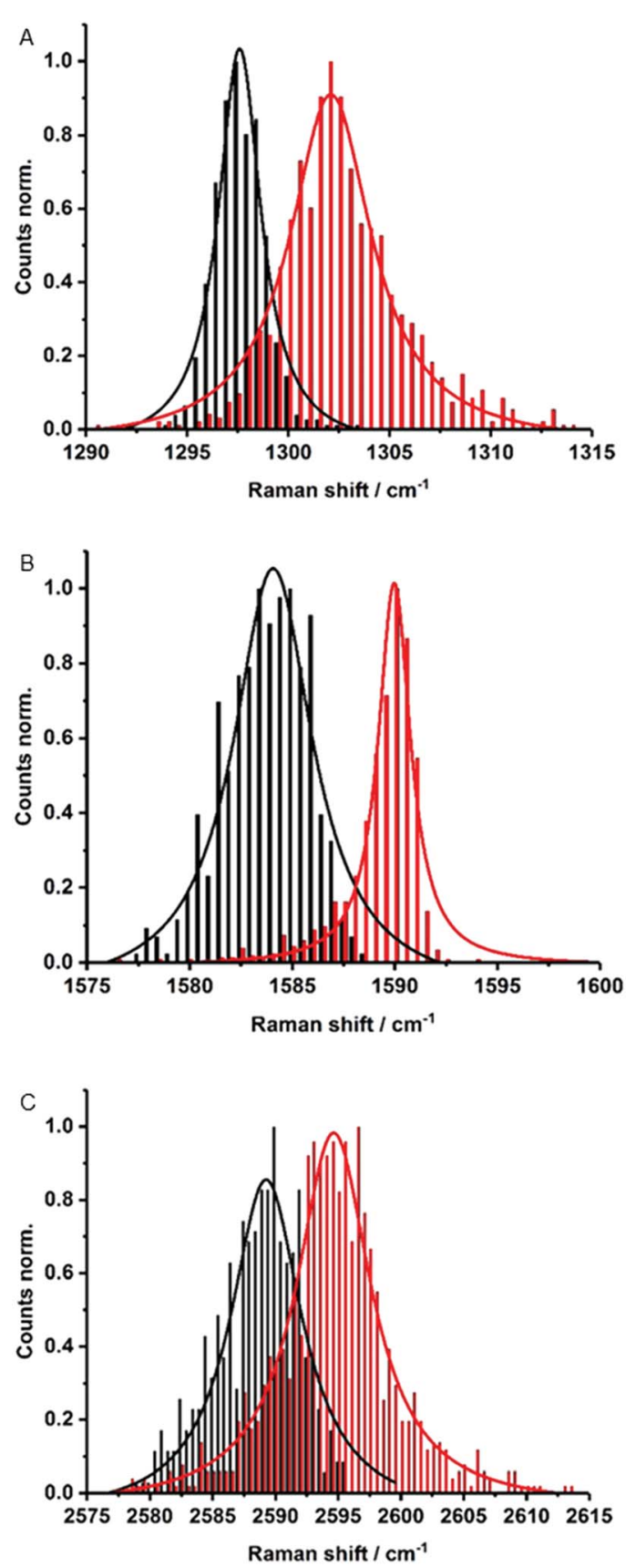

Fig. 4 Raman histograms of (A) D-mode, (B) G-mode, and (C) 2Dmode of drop-casted SWCNTs (black) and MINT-por (red) from methanol, with $633 \mathrm{~nm}$ laser excitation.
$2589 \mathrm{~cm}^{-1}$ for $(6,5)$-enriched SWCNTs to 1302, 1590 and $2595 \mathrm{~cm}^{-1}$ for MINT-por. For MINT-por the up-shifts indicate weak charge-transfer interactions, which relate to a donating of charge density from the porphyrin to the SWCNTs.

Raman spectroscopy is also a powerful tool to investigate chiral selectivity. To this end, the focus was on the low frequency radial breathing modes, which are unique for each SWCNT chirality (see Fig. S5†). Here, signals appear at about 255, 282 and $303 \mathrm{~cm}^{-1}$, which stem from $(7,6)-,(7,5)-$, and $(6,5)-$ SWCNTs, respectively. Well in line with the results from absorption and fluorescence measurements, the relative intensity of $(7,5)$-SWCNTs is higher in MINT-por than in the pristine sample of SWCNTs. Important is the fact that no significant changes were noted in the $I_{\mathrm{D}} / I_{\mathrm{G}}$ ratios when comparing $(6,5)$ enriched SWCNTs (0.12) with MINT-por (0.14). This confirms the absence of defect introduction during the functionalization.

Notable quenching in the fluorescence measurements prompted us to perform femtosecond transient absorption measurements - Fig. 5 and S6. $\dagger$ At first glance, the spectra of (6,5)-enriched SWCNTs and MINT-por are dominated by an immediate ground state bleaching of SWCNTs in the nIRregion. Hereby, the nIR bleachings mirror image the steady state absorption spectra. In stark contrast, contributions from the porphyrins in MINT-por in the visible region are not discernable as they are masked by the much stronger SWCNT
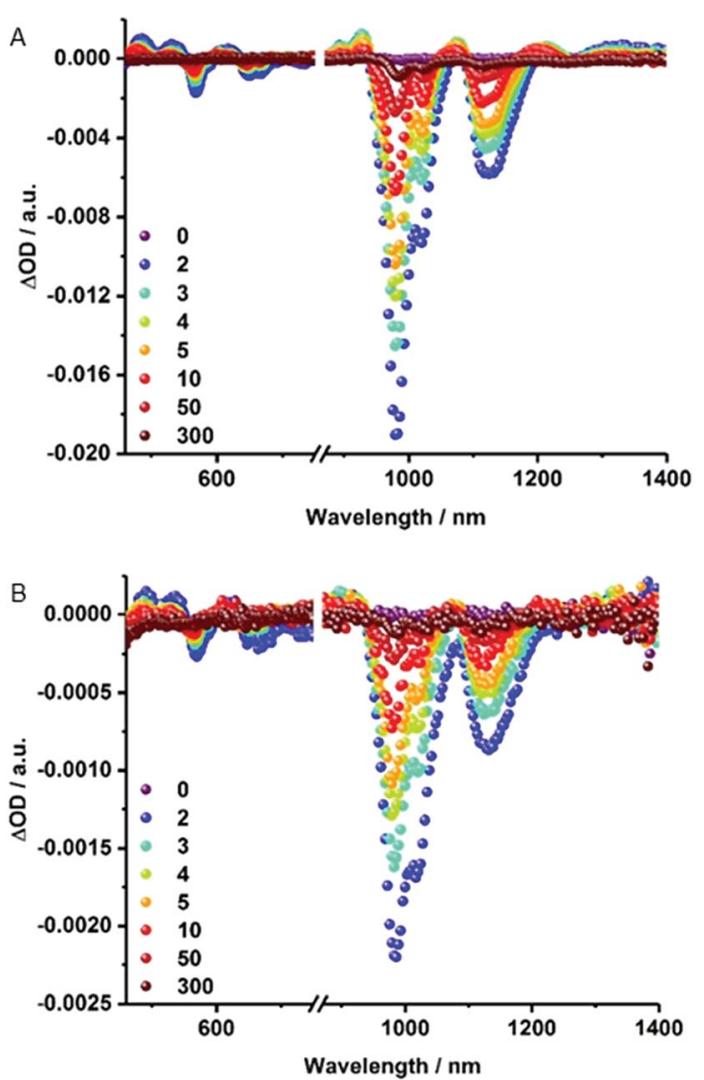

Fig. 5 Differential absorption spectra of (A) (6,5)-enriched SWCNTs and (B) MINT-por obtained upon femtosecond pump probe experiments following $420 \mathrm{~nm}$ laser excitation in $\mathrm{D}_{2} \mathrm{O} / \mathrm{SDBS}(1 \mathrm{wt} \%, \mathrm{rt})$ at several time delays between 0 and 300 ps. 
features. A closer look reveals that for $(6,5)$-enriched SWCNTs minima evolve at 568, 648, 667, 983, and $1121 \mathrm{~nm}$, while maxima appear at 484, 531, 612, 927, 1073, 1211, and >1300 nm. In MINT-por, the same features are discernable but red-shifted to $569,650,669,986$ and $1121 \mathrm{~nm}$ in the case of the minima and to $490,535,617,927,1076,1215$ and $>1300 \mathrm{~nm}$ in the case of the maxima. A three exponential fitting procedure of the ground state recovery of $(6,5)-,(7,5)-,(8,4)$ - and $(7,6)$-SWCNTs yielded three different lifetimes, which differ for each SWCNT chirality - Table S1. $\dagger$ In SWCNTs, the long-lived component relates to radiative exciton recombination, while the two short-lived components stem from interband- and intertube relaxations. In MINT-por, all lifetimes are markedly shorter. Interestingly, smaller diameter $(6,5)$ - and $(7,5)$-SWCNTs, which are subjective to the strongest fluorescence quenching, give rise to the fastest recovery. The long-lived component is decreased in, for example, $(6,5)$-SWCNTs by $32 \%$ and by $52 \%$ for $(7,5)$-SWCNTs. In stark contrast, in larger diameter $(8,4)$ - and $(7,6)$-SWCNTs the decrease of the long-lived component is only $15 \%$ and $12 \%$, respectively. ${ }^{54}$ We conclude that charge separation in MINT-por with 5 ps for $(6,5)$-SWCNT and 4 ps for $(7,5)$-SWCNT is followed by charge recombination with 78 and $81 \mathrm{ps,} \mathrm{respectively.} \mathrm{In} \mathrm{the}$ case of larger diameter SWCNTs both charge separation and recombination are less favored: the corresponding lifetimes are 4 and 72 ps for $(8,4)$ - as well as 3 and 52 ps for $(7,6)$-SWCNTs.

\section{Conclusions}

In the current study, we have documented the interfacing of porphyrin chromophores and SWCNTs via mechanical interlocking, through the synthesis of rotaxane-type architectures. Formation of MINTs is confirmed via adequate control experiments, analytical, spectroscopic and microscopic techniques. Besides TGA, the threading of the macrocycles with SWCNTs to form MINTs without introducing SWCNT defects was confirmed by means of AFM, AC-HRTEM and Raman spectroscopy. Moreover, from Raman and steady state absorption spectroscopy we concluded the selective functionalization of smaller diameter SWCNTs. In line with chiral selectivity, electronic interactions, which are intense between the porphyrin rings and SWCNTs of smaller diameters, are probably the most important factor. Transient absorption spectroscopy proved also to be very important to detect charge separation and recombination in MINT-por and to dissect the underlying dynamics. We are currently exploring the consequences that this entirely novel approach to connect porphyrins and SWCNTs has on applications such as catalysis, magnetism, and sensing. ${ }^{55,56}$

\section{Conflicts of interest}

There are no conflicts to declare.

\section{Acknowledgements}

Funding from the European Research Council (307609-StG), MINECO (CTQ2014-60541-P, FPU13/03371, JdC-2015-23531, CTQ2017-86060-P) and the Comunidad de Madrid (MAD2D-
CM S2013/MIT-3007) is gratefully acknowledged. IMDEA Nanociencia acknowledges support from the "Severo Ochoa" Programme for Centres of Excellence in R\&D (MINECO, Grant SEV-2016-0686). The computational work was supported by the Campus de Excelencia Internacional UAM+CSIC. We thank the Supercomputing and Bioinnovation Center (SCBI) of the University of Málaga (Spain) for their support and resources. We thank the National Centre for Electron Microscopy (ICTSCNME, Universidad Complutense) for electron microscopy facilities. P. W. M. acknowledges the Graduate School of Molecular Science for funding. This work was further supported by the Deutsche Forschungsgemeinschaft as part of the Excellence Cluster Engineering of Advanced Materials and SFB 953 Synthetic Carbon Allotropes as well as by the Bavarian State Government as part of the Solar Technologies go Hybrid initiative.

\section{Notes and references}

1 L.-L. Li and E. W.-G. Diau, Chem. Soc. Rev., 2013, 42, 291-304. 2 M. Ethirajan, Y. Chen, P. Joshi and R. K. Pandey, Chem. Soc. Rev., 2011, 40, 340-362.

3 J. A. A. W. Elemans, R. van Hameren, R. J. M. Nolte and A. E. Rowan, Adv. Mater., 2006, 18, 1251-1266.

4 J. L. Sessler and D. Seidel, Angew. Chem., Int. Ed., 2003, 42, 5134-5175.

5 D. M. Guldi, Chem. Soc. Rev., 2002, 31, 22-36.

6 T. Umeyama and H. Imahori, J. Phys. Chem. C, 2013, 117, 3195-3209.

7 D. M. Guldi and R. D. Costa, J. Phys. Chem. Lett., 2013, 4, 1489-1501.

8 M. F. L. De Volder, S. H. Tawfick, R. H. Baughman and A. J. Hart, Science, 2013, 339, 535-539.

9 N. Karousis, N. Tagmatarchis and D. Tasis, Chem. Rev., 2010, 110, 5366-5397.

10 S. F. Liu, A. R. Petty, G. T. Sazama and T. M. Swager, Angew. Chem., Int. Ed., 2015, 54, 6554-6557.

11 S. F. Liu, L. C. H. Moh and T. M. Swager, Chem. Mater., 2015, 27, 3560-3563.

12 A. Maurin and M. Robert, J. Am. Chem. Soc., 2016, 138, 24922495.

13 P.-J. Wei, G.-Q. Yu, Y. Naruta and J.-G. Liu, Angew. Chem., Int. Ed., 2014, 53, 6659-6663.

14 I. Hijazi, T. Bourgeteau, R. Cornut, A. Morozan, A. Filoramo, J. Leroy, V. Derycke, B. Jousselme and S. Campidelli, J. Am. Chem. Soc., 2014, 136, 6348-6354.

15 S. Jayakumar, H. Li, J. Chen and Q. Yang, ACS Appl. Mater. Interfaces, 2018, 10, 2546-2555.

16 Q. Zhong, V. V. Diev, S. T. Roberts, P. D. Antunez, R. L. Brutchey, S. E. Bradforth and M. E. Thompson, ACS Nano, 2013, 7, 3466-3475.

17 H. Imahori, T. Umeyama, K. Kurotobi and Y. Takano, Chem. Commun., 2012, 48, 4032-4045.

18 L. M. Arellano, M. Barrejon, H. B. Gobeze, M. J. GomezEscalonilla, J. L. G. Fierro, F. D'Souza and F. Langa, Nanoscale, 2017, 9, 7551-7558. 
19 G. Delport, F. Vialla, C. Roquelet, S. Campidelli, C. Voisin and J.-S. Lauret, Nano Lett., 2017, 17, 6778-6782.

20 T. Palacin, H. L. Khanh, B. Jousselme, P. Jegou, A. Filoramo, C. Ehli, D. M. Guldi and S. Campidelli, J. Am. Chem. Soc., 2009, 131, 15394-15402.

21 D. M. Guldi, H. Taieb, G. M. A. Rahman, N. Tagmatarchis and M. Prato, Adv. Mater., 2005, 17, 871-875.

22 D. M. Guldi, G. M. A. Rahman, M. Prato, N. Jux, S. Qin and W. Ford, Angew. Chem., Int. Ed., 2005, 44, 2015-2018.

23 G. Bottari, O. Trukhina, M. Ince and T. Torres, Coord. Chem. Rev., 2012, 256, 2453-2477.

24 F. D'Souza and O. Ito, Chem. Commun., 2009, 4913-4928, DOI: $10.1039 /$ b905753f.

25 S. Fukuzumi and T. Kojima, J. Mater. Chem., 2008, 18, 14271439.

26 L. Rodriguez-Pérez, S. Vela, C. Atienza and N. Martin, Org. Lett., 2017, 19, 4810-4813.

27 J. Lopez-Andarias, S. H. Mejias, T. Sakurai, W. Matsuda, S. Seki, F. Feixas, S. Osuna, C. Atienza, N. Martin and A. L. Cortajarena, Adv. Funct. Mater., 2017, 1704031.

28 C. Ehli, G. M. A. Rahman, N. Jux, D. Balbinot, D. M. Guldi, F. Paolucci, M. Marcaccio, D. Paolucci, M. Melle-Franco, F. Zerbetto, S. Campidelli and M. Prato, J. Am. Chem. Soc., 2006, 128, 11222-11231.

29 S. Chichak Kelly, A. Star, M. V. P. Altoé and J. F. Stoddart, Small, 2005, 1, 452-461.

30 G. Liu, F. Wang, S. Chaunchaiyakul, Y. Saito, A. K. Bauri, T. Kimura, Y. Kuwahara and N. Komatsu, J. Am. Chem. Soc., 2013, 135, 4805-4814.

31 A. F. M. M. Rahman, F. Wang, K. Matsuda, T. Kimura and N. Komatsu, Chem. Sci., 2011, 2, 862-867.

32 X. Peng, N. Komatsu, T. Kimura and A. Osuka, ACS Nano, 2008, 2, 2045-2050.

33 X. Peng, N. Komatsu, T. Kimura and A. Osuka, J. Am. Chem. Soc., 2007, 129, 15947-15953.

34 X. Peng, N. Komatsu, S. Bhattacharya, T. Shimawaki, S. Aonuma, T. Kimura and A. Osuka, Nat. Nanotechnol., 2007, 2, 361-365.

35 R. S. Forgan, J.-P. Sauvage and J. F. Stoddart, Chem. Rev., 2011, 111, 5434-5464.

36 J. F. Stoddart, Chem. Soc. Rev., 2009, 38, 1802-1820.

37 J. F. Stoddart, Angew. Chem., Int. Ed., 2017, 56, 11094-11125. 38 J.-P. Sauvage, Angew. Chem., Int. Ed., 2017, 56, 11080-11093. 39 D. A. Leigh, Angew. Chem., Int. Ed., 2016, 55, 14506-14508.

40 E. M. Pérez, Chem.-Eur. J., 2017, 23, 12681-12689.

41 A. Mann Florian, J. Horlebein, F. Meyer Nils, D. Meyer, F. Thomas and S. Kruss, Chem.-Eur. J., 2018, DOI: 10.1002/ chem.201800993.

42 K. Miki, K. Saiki, T. Umeyama, J. Baek, T. Noda, H. Imahori, Y. Sato, K. Suenaga and K. Ohe, Small, 2018, 14, 1800720.
43 S. Leret, Y. Pouillon, S. Casado, C. Navio, A. Rubio and E. M. Pérez, Chem. Sci., 2017, 8, 1927-1935.

44 A. Lopez-Moreno and E. M. Pérez, Chem. Commun., 2015, 51, 5421-5424.

45 A. de Juan, Y. Pouillon, L. Ruiz-Gonzalez, A. Torres-Pardo, S. Casado, N. Martin, A. Rubio and E. M. Pérez, Angew. Chem., Int. Ed., 2014, 53, 5394-5400.

46 A. López-Moreno, B. Nieto-Ortega, M. Moffa, A. de Juan, M. M. Bernal, J. P. Fernández-Blázquez, J. J. Vilatela, D. Pisignano and E. M. Pérez, ACS Nano, 2016, 10, 80128018.

47 E. Martinez-Perinan, A. de Juan, Y. Pouillon, C. Schierl, V. Strauss, N. Martin, A. Rubio, D. M. Guldi, E. Lorenzo and E. M. Pérez, Nanoscale, 2016, 8, 9254-9264.

48 R. Sure and S. Grimme, J. Comput. Chem., 2013, 34, 16721685.

49 A plot of the degree of functionalization $v s$. [U-por $]_{0}$ shows that the degree of functionalization does not have a linear relationship with the concentration of linear precursor, but instead shows a square hyperbolic shape, reminiscent of a $1: 1$ binding isotherm, reaching a maximum at about $30 \%$ (see Fig. S1 $\dagger$ ). Control experiments in which we mixed U-por with $(6,5)$-SWCNTs without Grubbs' catalyst, under otherwise identical conditions to the MINT reaction, yielded much lower functionalization (7\%).

50 A. de Juan, M. Mar Bernal and E. M. Pérez, ChemPlusChem, 2015, 80, 1153-1157.

51 These data taken together further prove that the supramolecular functionalization of the nanotubes by $\mathbf{U}$ por, mac-por and/or linear oligomers formed in situ is minoritary.

52 S. T. Skowron, T. W. Chamberlain, J. Biskupek, U. Kaiser, E. Besley and A. N. Khlobystov, Acc. Chem. Res., 2017, 50, 1797-1807.

53 In DMF rather than $\mathrm{D}_{2} \mathrm{O} / \mathrm{SDBS}$ a blue-shift of the $\mathrm{S}_{11}$ transitions for MINT-por relative to SWCNTs indicates a better debundling of SWCNTs.

54 Notably, we lack unambiguous proofs for the formation of the one-electron oxidized porphyrin, but we presume, as a sum of all spectroscopic evidence, charge transfer in small diameter SWCNTs. Here, the low intensity porphyrin transients, on one hand, and the strong SWCNT transients, on the other hand, are largely responsible for the lack of detection.

55 V. Sgobba, G. M. A. Rahman, D. M. Guldi, N. Jux, S. Campidelli and M. Prato, Adv. Mater., 2006, 18, 2264-2269.

56 D. M. Guldi and V. Sgobba, Chem. Commun., 2011, 47, 606610. 